\title{
The Value of Carotid Intima-Media Thickness in the Detection of Atherosclerosis in HIV (+) Patients Subclinical Atherosclerosis in HIV (+)
}

\author{
Selin Ozdemir ${ }^{1}$, Emre Ozdemir ${ }^{2}$, Bilge Birlik ${ }^{3}$ and Tuna Demirdal ${ }^{4}$ \\ ${ }^{1}$ Department of Infectious Diseases, Izmir Tire Government Hospital, Izmir, Turkey \\ ${ }^{2}$ Department of Cardiology, Izmir Katip Celebi University, Atatürk Training and Research Hospital, Izmir, Turkey \\ ${ }^{3}$ Department of Radiology, Izmir Ataturk Education and Research Hospital, Izmir, Turkey \\ ${ }^{4}$ Department of Infectious Diseases, Izmir Katip Celebi University, Atatürk Training and Research Hospital, Izmir, Turkey
}

\begin{abstract}
Background: To evaluate the role of the traditional risk scoring system (TRSS) in detecting subclinical atherosclerosis in HIV (+) patients.

Study Design: Cohort study.

Place and Duration of Study: Infectious Diseases Clinic, Izmir Katip Çelebi University, Atatürk Training and Research Hospital, from March 2017 to January 2018.

Methodology: The patient group was formed with 52 HIV (+) patients, aged 18-60 years, and a control group was formed with 52 HIV (-) healthy volunteers. For all groups, there was no comorbid diseases or family history. Diabetes mellitus, hypertension, chronic kidney disease and cardiovascular disease were excluded from the two groups. Carotid intima-media thickness (CIMT) measurements were performed with high resolution B mode Doppler USG and patients with subclinical atherosclerosis were identified by the presence of atheroma plaque.

Results: The median right CIMT measurement was $0.91(0.73-0.97) \mathrm{mm}$ and the median left CIMT was $0.90(0.73-0.98) \mathrm{mm}$ in HIV (+) patients. The median values of CIMT on right and left sides in the control group were $0.77(0.67-0.81) \mathrm{mm}$ and $0.76(0.70-0.81) \mathrm{mm}$. Atheroma plaque was detected in $13.5 \%$ of the HIV (+) patients and in none of the control group. Subclinical atherosclerosis was found in $51.9 \%$ of HIV (+) patients and this rate was $7.7 \%$ in the HIV $(-)$ group $(p<0.001)$. There was a weak correlation between CIMT and TRSS.

Conclusion: In this study, the scoring systems (Framingham, ACC/AHA CVHRS) that determine the risk of cardiovascular disease recommended in current practice and the results of CIMT measurements were not found to be compatible. The development of new scoring systems including CIMT testing for the determination of this risk will open important new horizons.
\end{abstract}

Key Words: AIDS, Cardiovascular disease, Subclinical atherosclerosis, CIMT.

How to cite this article: Ozdemir S, Ozdemir E, Birlik B, Demirdal T. The Value of Carotid Intima-Media Thickness in the Detection of Atherosclerosis in HIV (+) Patients Subclinical Atherosclerosis in HIV (+). J Coll Physicians Surg Pak 2021; 31(07):759-764.

\section{INTRODUCTION}

With the new-generation antiretroviral treatment (ART) options currently used in the treatment of HIV (human immunodeficiency virus), infected patients' life expectancy is prolonged; but chronic problems that may cause, mortality may occur, if not well managed. Individuals with HIV infection are at high risk for atherosclerosis and myocardial infarction. ${ }^{1}$ However, most of the nearly 40 million people worldwide with HIV live in developing countries and approximately 6 million of them need immediate ART. ${ }^{2}$

Correspondence to: Dr. Emre Ozdemir, Department of Cardiology, Izmir Katip Celebi University, Atatürk Training and Research Hospital, Izmir, Turkey E-mail: emreozdemir27@yahoo.com.tr

Received: October 07, 2020; Revised: May 28, 2021; Accepted: June 23, 2021

DOI: https://doi.org/10.29271/jcpsp.2021.07.759
In the pathogenesis of atherosclerosis in HIV (+) patients, the social risks of HIV $(+)$, the persistent inflammation caused by HIV infection and the side effects of ART used, can help explain this relationship. ${ }^{1}$ In treatment-independent dyslipidemia in HIV (+) patients, low density lipoprotein (LDL) and high density lipoprotein (HDL) levels decrease and triglyceride (TG) and very low density lipoprotein (VLDL) levels may increase. Following treatment, TG, total cholesterol, and LDL levels may also increase. $^{3}$

Exercise ECG (electrocardiogram), myocardial perfusion scintigraphy, coronary computed tomography angiography, conventional coronary angiography and carotid intima-media thickness (CIMT) measurements have been shown to be economical and non-invasive methods for the prediction of the risk of cardiovascular disease and atherosclerosis. ${ }^{4}$

The cardiovascular risk (CVR) of HIV (+) patients can be evaluated using the Framingham risk score (FRS-CVD-10), the Amer- 
ican Society of Cardiology / American Heart Association cardiovascular disease risk score (ACC/AHA CVDRS) and the HIV-infected cohort cardiovascular disease risk score (D: A: DCVHRS). However, these risk scores may over- or under-estimate the actual cardiovascular events for $\mathrm{HIV}(+)$ patients. ${ }^{5}$

The aim of this study was to evaluate the compatibility of subclinical atherosclerosis and scoring systems for CVR through determination of CIMT.

\section{METHODOLOGY}

This cohort study was conducted with 52 HIV (+) patients, aged 18-60 years, who were followed up in the Infectious Diseases Clinic of Izmir Katip Çelebi University, Atatürk Training and Research Hospital and a control group was formed with 52 HIV (-) healthy volunteers. No patient or control group subject had any known diabetes mellitus, hypertension, chronic kidney disease orcardiovascular disease.

Clinical information was obtained and dietary and sports habits were questioned, then systolic / diastolic blood pressure and waist circumference were measured. Complementary laboratory tests were performed during outpatient clinic visits, including blood glucose, creatinine, blood urea nitrogen, Tc (total cholesterol), LDL, HDL, TG, AST (aspartate transaminase), ALT (alanine transaminase) and CRP (c-reactive protein). Regular exercise of $\geq 20$ minutes $\geq 2$ days a week was considered as an 'exercise habit'. Dietary habits were scored with respect to high carbohydrate consumption, high fatty food consumption, fast food consumption habits and salt consumption habits (very salty / less salty / no salt). A score of $<8$ out of a total of 9 points was accepted as 'bad dietary habit', and scores of 8 and 9 as 'good dietary habits'. Body mass index (BMI) was calculated from the height and weight values, and classified as $18.5-24.9 \mathrm{Kgs} / \mathrm{m}^{2}=$ normal weight, $25-29.9 \mathrm{Kgs} / \mathrm{m}^{2}=$ overweight, and $\geq 30 \mathrm{Kgs} / \mathrm{m}^{2}$ = obese. HIV-related risk factors were recorded by investigating the CD4 count, viral load, duration of the disease, and type and duration of antiretroviral therapy. The Framingham risk score (FRS) and ACC/AHA CVDRS score were calculated.

CMIT was measured by a specialist radiologist from the Radiodiagnostic Department of the Hospital, blinded to the study groups, using a high-resolution B-mode ultrasonography (USG) device (Applio 300 Ultrasound and 11L4 linear probe, Canon). The measurements were performed manually from the right and left common carotid artery three times, and the mean thickness value was calculated. CIMT $>0.9 \mathrm{~mm}$ or atheroma plaque was accepted as 'subclinical atherosclerosis' and high-risk for cardiovascular disease. CIMT $>1.5 \mathrm{~mm}$, or echogenicities extending to the lumen $\geq 0.5 \mathrm{~mm}$ or echogenicities exceeding $50 \%$ of the adjacent CMIT in the extending vessel and extending into the lumen

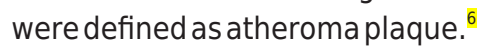

Approval for the study was granted by the local ethics committee. Informed consent was obtained from all study participants.

Data obtained in the study were analysed statistically using SPSS version 21 software (SPSSInc., Chicago, IL, USA). Continuousvariables were expressed as mean \pm standard deviation and median and interquartile range (IQR: 25th percentile-75th percentile), and categorical variables were expressed as the number of patients and percentage (\%). Student's t-test was used for the analysis of normally distributed variables and Mann-Whitney Utest for variables not showing normal distribution. Chi-square or Fisher's Exact test were used for the comparison of categorical variables between groups. ROC analysis was used to show the connection between subclinical atherosclerosis, detected by CIMT, and riskscores for clinical sensitivity and specificity values. Values of $p<0.05$ were considered statistically significant.

\section{RESULTS}

The study enrolled a total of 55 patients admitted to the polyclinic between March 2017 and January 2018, who met the study inclusion criteria. Three patients were excluded from the study because they did not take the CIMT measurement test required by the study, so evaluation was made from the data of $52 \mathrm{HIV} \mathrm{(+)} \mathrm{patients.} \mathrm{A} \mathrm{control} \mathrm{group} \mathrm{was} \mathrm{formed} \mathrm{of} 52$ HIV (-) healthy volunteers. Thus the cohort comprised a total of 104 subjects.

The mean age of the HIV ( + ) study group was $43.3 \pm 10.0$ years, and the mean age of the control group was $43 \pm 10.2$ years. In both the study and control groups, $30.8 \%(n=16)$ were females and $69.2 \%$ were males (Table I).

The median body mass index of HIV (+) patients was 24.5 $(22.7-29.2) \mathrm{kg} / \mathrm{m}^{2}$. The median BMl of the patients in the control group was $26.2(24.0-29.8) \mathrm{Kg} / \mathrm{m}^{2}$. A total of $24(46.2 \%)$ patients (obese and overweight) in the study group and $34(65.4 \%)$ in the control group had BMI values above normal $\left(>24.9 \mathrm{~kg} / \mathrm{m}^{2}\right)$. The mean waist circumference was $93.3 \pm 12.3 \mathrm{~cm}$ in the HIV (+) patient group and $88.6 \pm 13.0 \mathrm{~cm}$ in the control group. The median systolic/diastolic blood pressure values were $115.0 / 80.0 \mathrm{mmHg}$ in the HIV (+) group, and $120.0 / 80.0 \mathrm{mmHg}$ in the control group (Tablel).

A bad dietary habit was determined in 41 (78.8\%) HIV (+) patients and in $34(65.4 \%)$ of the control group. There was no statistically significant difference in HIV (+) patients compared to the control group $(p=0.126)$. A regular exercise habit was recorded for $38.5 \%(n=20)$ of HIV $(+)$ patients, and $15.4 \%(n=8)$ of the control group. A regular exercise habit was seen to be statistically significantly greaterin $\mathrm{HIV}(+)$ patients $(p=0.008)$.

The median creatinine level was $0.82(0.77-0.93) \mathrm{mg} / \mathrm{dl}$ in HIV $(+)$ patients, and $0.76(0.70-0.84) \mathrm{mg} / \mathrm{dl}$ in the control group. This increase in creatinine values in the HIV (+) patient group was statistically significant $(p=0.005)$. The median blood triglyceride and the mean HDLc levels were 144.5 (107.8-222.5) $\mathrm{mg} / \mathrm{dL}$ and $42.1 \pm 9.4 \mathrm{mg} / \mathrm{dL}$ in the HIV (+) patient group and $124.0(89.5-167.8) \mathrm{mg} / \mathrm{dL}$ and $48.5 \pm 10.5 \mathrm{mg} / \mathrm{dL}$ in the control group, respectively. There was a significant difference between TG and HDL values between the two groups $(p=0.040$ and $p=$ 0.001 , respectively). In accordance with this, a statistically significant difference was observed in the calculated TG / HDL ratio $(p=0.004$, Table II). 
Table I: Demographic characteristics of the study and control groups.

\begin{tabular}{|l|c|c|}
\hline & HIV (+) patients & HIV(-) patients \\
\hline Mean age \pm SD (years) & $43.3 \pm 10.0$ & $43 \pm 10.2$ \\
\hline Male (n,\%) & $36(69.2 \%)$ & $36(69.2 \%)$ \\
\hline Median(IQR) BMI $\left(\mathrm{kg} / \mathrm{m}^{2}\right)$ & $24.5(22.7-29.2)$ & $26.2(24.0-29.8)$ \\
\hline Overweight and obese patients (n,\%) & $24(46.2 \%)$ & $34(65.4 \%)$ \\
\hline Mean waist circumference (cm) \pm SD & $93.3 \pm 12.3$ & $88.6 \pm 13.0$ \\
\hline Median(IQR) SBP (mmHg) & $115.0(110.0-120.0)$ & $120.0(110.0-120.0)$ \\
\hline Median(IQR) DBP (mmH) & $80.0(70.0-80.0)$ & $80.0(70.0-80.0)$ \\
\hline SD: Standard deviation; BMl: Body mass index; SBP: Systolic blood presure; DBP: Diastolic blood presure; IQR: 25th percentile-75th percentile (Interquartile range). \\
\hline
\end{tabular}

Table II: Laboratory measurement data.

\begin{tabular}{|c|c|c|c|}
\hline Variable & HIV (+) patients & HIV (-) patients & p-value \\
\hline Mean Blood Glucose \pm SD $(\mathrm{mg} / \mathrm{dl})$ & $95.9 \pm 19$ & $97.6 \pm 13.3$ & 0.592 \\
\hline Median(IQR) BUN (mg/dl) & $13(11.0-15.0)$ & $12(10.0-14.8)$ & 0.678 \\
\hline Median(IQR) Creatinine (mg/dl) & $0.82(0.77-0.93)$ & $0.76(0.70-0.84)$ & 0.005 \\
\hline Median(IQR) AST (IU/ml) & $20(16.0-22.0)$ & $19(16.0-25.5)$ & 0.889 \\
\hline Median(IQR) ALT (IU/ml) & 19(14.0-27.0) & $22.5(16.0-33.5)$ & 0.276 \\
\hline Median(IQR) CRP (mg/dl) & $0.15(0.06-0.35)$ & $0.18(0.02-0.33)$ & 0.571 \\
\hline Mean Tc $\pm S D(m g / d l)$ & $202.0 \pm 47.1$ & $197.6 \pm 39.5$ & 0.605 \\
\hline Median(IQR) TG (mg/dl) & 144.5(107.8-222.5) & $124.0(89.5-167.8)$ & 0.040 \\
\hline Mean LDL $\pm \mathrm{SD}(\mathrm{mg} / \mathrm{dl})$ & $123.8 \pm 42.3$ & $124.1 \pm 36.2$ & 0.970 \\
\hline Mean HDL $\pm \mathrm{SD}(\mathrm{mg} / \mathrm{dl})$ & $42.1 \pm 9.4$ & $48.5 \pm 10.5$ & 0.001 \\
\hline TG/HDL ratio & $0.58 \pm 0.27$ & $0.42 \pm 0.27$ & 0.004 \\
\hline Median(IQR) Right CIMT (mm) & $0.91(0.73-0.97)$ & $0.77(0.67-0.81)$ & 0.001 \\
\hline Median(IQR) Left CIMT (mm) & $0.90(0.73-0.98)$ & $0.76(0.70-0.81)$ & 0.001 \\
\hline Atheroma plaque $\%(n)$ & $13.5 \%(7)$ & $0 \%(0)$ & 0.013 \\
\hline Subclinical atherosclerosis\% (n) & $51.9 \%(27)$ & $7.7 \%(4)$ & $<0.001$ \\
\hline
\end{tabular}

Table III: The relationship of the groups with cardiovascular disease risk scores and biochemical parameters according to the presence of subclinical atherosclerosis.

\begin{tabular}{|c|c|c|c|}
\hline & $\begin{array}{l}\text { Subclinical atherosclerosis } \\
(\text { CIMT>0.9 mm) }\end{array}$ & $\begin{array}{l}\text { Normal CIMT } \\
(\mathrm{CIMT} \leq 0.9 \mathrm{~mm})\end{array}$ & p-value \\
\hline FRS-CVD-10<10\% $(n=39)^{*}$ & $46.2 \%(n=18)$ & $53.8 \%(n=21)$ & 0.138 \\
\hline ACC / AHA CVDRS $<7.5 \%(n=22) * *$ & $59.1 \%(n=13)$ & $40.9 \%(n=9)$ & 0.258 \\
\hline Mean Tc $\pm S D(m g / d L)$ & $216.9 \pm 51.0$ & $185.9 \pm 37.1$ & 0.016 \\
\hline Mean LDL $\pm \mathrm{SD}(\mathrm{mg} / \mathrm{dL})$ & $138.1 \pm 42.4$ & $108.3 \pm 37.1$ & 0.011 \\
\hline Mean HDL $\pm S D(m g / d L)$ & $42.3 \pm 9.8$ & $41.9 \pm 9.1$ & 0.898 \\
\hline Mean TG $\pm \mathrm{SD}(\mathrm{mg} / \mathrm{dL})$ & $182.9 \pm 92.3$ & 172. $2 \pm 98.1$ & 0.685 \\
\hline Median (IQR) CRP mg/dL & $0.23(0.120-0.410)$ & $0.090(0.040-0.185)$ & 0.009 \\
\hline
\end{tabular}

The mean measurement of right CIMT in HIV $(+)$ patients was $0.85 \pm 0.16 \mathrm{~mm}$ and the median value was 0.91 (0.73-0.97) $\mathrm{mm}$, the left CIMT was a mean of $0.88 \pm 0.20$ $\mathrm{mm}$ and a median of $0.90(0.73-0.98) \mathrm{mm}$. In the control group, the mean value of right / left CIMT was $0.76 \pm 0.12$ / $0.76 \pm 0.12 \mathrm{~mm}$, and the median values were 0.77 (0.67-0.81) and $0.76(0.70-0.81) \mathrm{mm}$, respectively. The CIMT values for both sides were statistically significantly increased in the HIV $(+)$ patients $(p=0.001)$. Subclinical atherosclerosis (CIMT >0.9 mm) was detected in $51.9 \%$ $(n=27)$ of HIV $(+)$ patients, and in $4(7.7 \%)$ in the control group. Subclinical atherosclerosis was significantly higher in
HIV (+) patients than the control group $(p<0.001)$. Atheroma plaque was detected in $13.5 \%(n=7)$ in the HIV (+) group, and in none in the control group (Table II).

FRS-CVD-10 for patients younger than 30 years $(n=3)$ and ACC / AHA CVDRS for patients younger than 40 years $(n=$ 19) could not be calculated. FRS-CVD-10 was calculated for 49 patients; $46.2 \%(n=18)$ of these patients had CIMT $>0.9$ and $53.8 \%(n=21)$ of these patients had CIMT $\geq 0.9$ in FRSCVD-10 $<10 \%$ group. There was no statistical difference $(p=0.138)$. AHA/ACC CVDRS was calculated for 33 patients; $59 \%(n=13)$ of these patients had CIMT $>0.9$ and $41 \%(n=9)$ of these patients had CIMT $\geq 0.9$ in the CVDRS $<7.5 \%$ group. 
There was no statistical difference ( $p=0.258$, Table III).

The median HIV (+) diagnosis time for patients was 50.0 (20.0-87.8) months. The number of patients receiving antiretroviral treatment (ART) was 51(98.1\%), and the median duration of ART was 36.0 (12.0-72.0) months. Mean CD4 was $664.1 \pm 342.9$ cells $/ \mathrm{mm}^{3}$, and mean CD8 was $829.7 \pm 342.6$ cells $/ \mathrm{mm}^{3}$. The most common ART regimens were: protease inhibitor regimen $(49 \%, n=25)$, integrase inhibitor regimen $(35.3 \%, n=18)$ and non-nucleoside reverse transcriptase inhibitor regimen $(15.7 \%, \mathrm{n}=8)$.

The correlation between the presence of subclinical atherosclerosis and high-risk patients (FRS-CVD-10>10\%) was evaluated by ROC analysis. There was a poor correlation between subclinical atherosclerosis and FRS-CVD-10>10\% patients (AUROC:0.696; 95\%Cl $0.578-0.813 ; p=0.002$ ).

\section{DISCUSSION}

With more effective treatment of HIV infection, morbidity and mortality related to HIV has decreased and mortality rates due to chronic diseases have increased. Cardiovascular diseases (CVD) are the major reason for mortality in the chronic phase of HIV (+). The incidence of myocardial infarction in HIV $(+)$ patients is 1.5 -fold greater compared to HIV () individuals. In HIV (+) patients, hypertension, smoking, insulin resistance, hypercholesterolemia and smoking, which are traditional risk factors for cardiovascular disease, are more common than in the healthy population. ${ }^{7,8}$

The smoking history of the patients in the control group was $34.6 \%$, similar to the smoking rate in Turkey; ${ }^{9}$ whereas, $69.2 \%$ of the HIV (+) patients had a history of smoking. Data on BMI in HIV-infected patients were examined and various studies have found that the prevalence of overweight or obesity ranged from $10.7 \%$ to $50 \% .{ }^{10}$ In the current study, the rate of overweight or obese patients was found to be $46.2 \%$ in the HIV (+) group, and $65.4 \%$ in the control group, which was consistent with previous findings in the literature. $^{11}$

Less than half of the HIV (+) patients (38.5\%) had regular exercise (at least 20 minutes / day physical activity for at least two days a week). However, the rate of regular exercise was $15.4 \%$ in the control group selected from the healthy population. The fact that this ratio was higher in HIV $(+)$ patients compared to the society average can be attributed to the fact that during outpatient clinic visits, they are encouraged and informed by physicians about the necessity for and benefits of regular physical activity.

HIV virus is known to affect the kidneys, and in addition to the virus, ART also contributes to side-effects. According to the study of Banda et al. in this study, serum creatinine levels were significantly higher in HIV (+) patients (0.82 vs. $0.76 ; p<0.05)^{12}$
HIV-induced immune reactions and antiretroviral therapy may exacerbate dyslipidemia. Tc, TG and LDL values may increase in patients receiving treatment and HDL may decrease. ${ }^{13}$ In the current study, TG levels were significantly increased in HIV (+) patients (144.5 vs. $124.0 \mathrm{mg} / \mathrm{dL}$, $\mathrm{p}=0.040)$ and HDL levels were significantly decreased (42.1 \pm 9.4 and $48.5 \pm 10.5 \mathrm{mg} / \mathrm{dL} ; \mathrm{p}=0.001)$. Tc $(202.0 \pm$ 47.1-197.6 \pm 39.5) and LDL (123.8 \pm 42.3-124.1 436.2$)$ values were similar in both groups. Similar LDL and Tc levels can be explained by the fact that the duration of treatment of the HIV (+) patient group was shorter than in other studies (median 36 months).

The use of CIMT measurement is recommended in asymptomatic patients to determine increased risk of cardiovascular disease. ${ }^{14}$ In the current study, CIMT measurements were taken in both groups to investigate whether HIV (+) patients are at increased cardiovascular risk, for which medical and social measures can be taken. The median values of both right and left CIMT were significantly increased in the HIV (+) patient group compared to the HIV negative group (right: 0.91 vs 0.77 and left: 0.90 vs 0.76 , both $p=0.001$ ). The presence of atheroma plaque was detected in $13.5 \%$ $(n=7)$ of HIV $(+)$ patients, and not detected in any of the HIV (-) group. When similar studies in the literature were examined, CIMT measurements were found to be significantly increased in HIV (+) patients. ${ }^{15}$

In a study by Mosepele et al., HIV (+) patients were compared with an HIV (-) control group and no significant difference was found between the groups with respect to the CIMT measurements, but atheroma plaque was determined at a significantly higher rate in the $\operatorname{HIV}(+)$ group. ${ }^{16}$ Hsue et al. also reported that even after adjusting for traditional risk factors, a higher CIMT measurement value was found in HIV (+) patients. ${ }^{17}$ In the current study, although the mean age was the same in both groups, a significant difference was found in terms of CIMT measurements ( $p=0.001$, Table II).

Patients with CIMT $>0.9 \mathrm{~mm}$ and/or atheroma plaque have been categorised as 'subclinical atherosclerosis'. De Socio et al. detected subclinical atherosclerosis at a rate of $41 \%$ at HIV (+) patients. ${ }^{18}$ In the current study, subclinical atherosclerosis was determined in $51.9 \%(n=27)$ of the HIV $(+)$ patient group and in $7.7 \%(n=2)$ of the control group. The rate of subclinical atherosclerosis of $51.9 \%$ was higher than in other studies in the literature.

According to this study, in a subgroup evaluation of 279 HIV (+) patients in Brazil, atherosclerosis was found to be associated with age and BMI, but gender and smoking habit were unrelated. ${ }^{19}$

Current guidelines for cardiovascular risk management in HIV (+) patients recommend to determine the possible risk using the FRS-CVD-10 and ACC / AHA CVDRS. ${ }^{13}$ However, these scores may be insufficient to determine the actual risk 
in the HIV population. ${ }^{20,21}$ In a study evaluating 84 HIV (+) patients with a low Framingham risk score (median FRS: $1 \%)$, the prevalence of subclinical carotid atherosclerosis was found to be high $(21 \%)$ and it was stated that these patients, evaluated according to FRS, were overlooked and did not receive primary prophylaxis for cardiovascular events. ${ }^{22}$ In the current study, there were 26 HIV (+) patients with FRS-CVD-10 calculated, who had subclinical atherosclerosis, and only $30.8 \%(n=8)$ patients in the subclinical atherosclerosis group were at moderate and high risk $(\geq 10 \%)$, while $69.2 \%(n=18)$ were at low risk $(<10 \%)$, according to the the FRS-CVD-10. There were 39 patients with a total FRS-CVD- $10<10 \%$ in the HIV (+) group, with subclinical atherosclerosis present in $46.2 \%$ of these patients with a low risk according to the FRS-CVD-10 (Table III). Correlation analysis showed a weak correlation between the CIMT measured values and the calculated FRS-CVD-10 scores, with a sensitivity of $30 \%$ for the presence of subclinical atherosclerosis. According to this, it was seen as a possible problem that patients who were found to be at low risk with FRS-CVD-10 could be overlooked and the necessary precautions and treatments would not be implemented.

The cardiovascular disease risk score (DAD CVDRS) is a scoring system that takes into account the risks associated with the use of ART in the HIV (+) patient population. Thompson-Paul et al. compared the SCORE and DAD CVD risk scores known as ASCVD and FRS, and European scores in the HIV (+) population, and found that the FRS and DAD CVD risk scores were calculated and no superiority was found for either scoring system. The positive predictive value of both scoring systems was moderate. ${ }^{23}$

In the current study, the mean values of Tc (216.9 $\pm 51-185.9$ $\pm 37.1) \mathrm{mm}$ and $\mathrm{LDL}(138.1 \pm 42.4-108.3 \pm 37.1) \mathrm{mm}$ were significantly increased in the group with subclinical atherosclerosis $(p=0.016-0.011)$. TG levels showed a mild increase $(182.9 \pm 92.3$ vs.172.2 \pm 98.1$) \mathrm{mm}$, while HDL levels were similar (Table III). This result may also be related to the fact that patients exposed to ART for a longer time (>24 months) were more likely to be in the subclinical atherosclerosis group. Increased CRP levels have been associated with a slower immunological response and increased risk of cardiovaskular disease. ${ }^{24}$ In the current study, CRP levels were found to be significantly increased in the subclinical atherosclerosis group ( $p=0.009$, Table III).

\section{CONCLUSION}

Subclinical atherosclerosis may be present in patients with low risk scores according to FRS-CVD-10 and ACC / AHA CVDRS. Considering the CIMT cut-off value as 0.9 , the use of CIMT for the same purpose in HIV (+) patients is recommended for ease of use, low cost and reproducibility. Therefore, the creation of a more powerful scoring system including CIMT measurements could lead to more effective results in determining cardiovascular risk in HIV $(+)$ patients.

\section{ETHICAL APPROVAL:}

Approval for the study was granted by the Non-Interventional Ethics Committee, Izmir Katip Çelebi University (Date: 22.02.2017, No. 49), prior to initiation of the study.

\section{PATIENTS' CONSENT:}

Patients' consents were obtained for the study.

\section{CONFLICT OF INTEREST:}

The authors declared no conflict of interest.

\section{AUTHORS' CONTRIBUTION:}

SO: Design of work, analysis.

EO: Drafting the work, writing.

$\mathrm{BB}$ : Interpretation of data for the work.

TD: Critical revision.

\section{REFERENCES}

1. Stein JH, Hsue PY. Inflammation, immune activation, and CVD risk in individuals with HIV infection. Jama 2012; 308(4):405-6. doi: 10.1001/jama.2012.8488.

2. Tahir NB, Uddin QT. Treatment adherence and outcomes of antiretroviral agents in HIV positive patients. J Coll Phys Surg Pak 2014; 24(9): 645-8.

3. Ssinabulya I, Kayima J, Longenecker C, Luwedde M, Semitala F, Kambugu A, et al. Subclinical atherosclerosis among HIV-infected adults attending HIV/AIDS care at two large ambulatory HIV clinics in Uganda. PloS one 2014; 9(2):e89537. doi: 10.1371/journal.pone.0089537.

4. Den Ruijter HM, Peters SA, Anderson TJ, Britton AR, Dekker $J M$, Eijkemans MJ, et al. Common carotid intima-media thickness measurements in cardiovascular risk prediction: A meta-analysis. Jama 2012; 308(8):796-803. doi: 10.1001/ jama.2012.9630.

5. Siontis GC, Tzoulaki I, Siontis KC, Ioannidis JP. Comparisons of established risk prediction models for cardiovascular disease: Systematic review. Bmj 2012; 344:e3318. doi: 10.1136/bmj.e3318.

6. Stein JH, Korcarz CE, Hurst RT, Lonn E, Kendall CB, Mohler $E R$, et al. Use of carotid ultrasound to identify subclinical vascular disease and evaluate cardiovascular disease risk: A consensus statement from the american society of echocardiography carotid Intima-media thickness task force endorsed by the society for vascular medicine. J Am Soc Echocardiogr 2008; 21(2):93-111. doi: 10.1016/j.echo. 2007.11.011.

7. Finegold JA, Asaria P, Francis DP. Mortality from ischaemic heart disease by country, region, and age: Statistics from World Health Organisation and United Nations. Int J Cardiol 2013; 168(2):934-45. doi: 10.1016/j.ijcard.2012.10.046.

8. Martin-Iguacel R, Llibre J, Friis-Moller N. Risk of cardiovascular disease in an aging HIV population: Where are we now? Current HIV/AIDS Reports 2015; 12(4):375-87. doi: 10.1007/s11904-015-0284-6.

9. Özer N, Kılıçkap M, Tokgözoğlu L, Göksülük H, Karaaslan D, Kayıkçıoğlu M, et al. Data on smoking in Turkey: Systematic review, meta-analysis and meta-regression of epi-demiological studies on cardiovascular risk factors. Arch Turk Soc 
Cardiol 2018; 46(7);602-12. doi: 10.5543/tkda.2018.85349.

10. Mashinya F, Alberts M, Colebunders R. Assessment of cardiovascular risk factors in people with HIV infection treated with ART in rural South Africa: A cross sectional study. AIDS Research And Therapy 2015; 12(1):42. doi: 10.1186/s12981-015-0083-6.

11. Oguz A, Temizhan A, Abaci A, Kozan Ö, Erol Ç, Öngen Z, et al. Obesity and abdominal obesity; an alarming challenge for cardio-metabolic risk in Turkish adults. Anatolian J Cardioogy 2008; 8(6):401.

12. Banda J, Mweemba A, Siziya S, Andrews B, Shabir L. Prevalence and factors associated with renal dysfunction in HIV positive and negative adults at the University teaching Hospital, in Lusaka. Med J Zambia 2014; 41(4):168-73.

13. Cahn P, Leite O, Rosales A, Cabello R, Alvarez C, Seas C, et al. Metabolic profile and cardiovascular risk factors among Latin American HIV-infected patients receiving HAART. Brazilian Journal of Infectious Diseases 2010; 14(2):158-66. doi: 10.1590/s1413-86702010000200008.

14. Bots ML, Evans GW, Tegeler CH, Meijer R. Carotid intima-media thickness measurements: Relations with atherosclerosis, risk of cardiovascular disease and application in randomised controlled trials. Chinese Med J 2016; 129(2):215. doi: 10.4103/0366-6999.173500.

15. Kwiatkowska W, Drelichowska-Durawa J, Czamecki M, Gasiorowski J, et al. Subclinical carotid atherosclerosis and cardiovascularrisk factors in HIV-infected patients. Postepy Hig Med Dosw (online) 2011; 65:770-83. doi: 10.5604/ 17322693.967075.

16. Mosepele M, Hemphill LC, Moloi W, Moyo S, Nkele I, Makhema J, et al. Pre-clinical carotid atherosclerosis and sCD163 among virally suppressed HIV patients in Botswana compared with uninfected controls. PloS One 2017; 12(6):e0179994. doi: 10.1371/journal.pone.0179994.

17. Hsue PY, Ordovas K, Lee T, Reddy G, Gotway M, Schnell A, et al. Carotid Intima-media thickness among human ımmun- odeficiency virus-ınfected patients without coronary calcium. Am J Cardiol 2012; 109(5):742-7. doi: 10.1016 /j.amjcard.2011.10.036.

18. De Socio G, Martinelli C, Ricci E, Orofino G, Valsecchi L, Vitiello $P$, et al. Relations between cardiovascular risk estimates and subclinical atherosclerosis in naïve HIV patients: Results from the HERMES study. Int J STD AIDS 2010; 21(4):267-72. doi: 10.1258/ijsa.2009.009165.

19. Salmazo PS, Bazan SGZ, Shiraishi FG, Bazan R, Okoshi K, Hueb JC. Frequency of subclinical atherosclerosis in Brazilian HIV-Infected patients. Arquivos Brasileiros de Cardiol Ahead 2018; 110(5):402-10. doi: 10.5935/abc. 20180058.

20. Law M, Friis-Møller N, El-Sadr W, Weber R, Reiss P, D'Arminio Monforte $A$, et al. The use of the framingham equation to predict myocardial infarctions in HIV-infected patients: Comparison with observed events in the D: A: D Study. HIV Med 2006; 7(4):218-30. doi: 10.1111/j.1468- 1293. 2006.00362.x.

21. Regan S, Meigs JB, Massaro J, D'Agostino RB, Grinspoon SK, Triant VA, editors. Evaluation of the ACC/AHA CVD risk prediction algorithm among HIV-infected patients. Conference on retroviruses and opportunistic infections; 2015.

22. León R, Reus S, López N, Portilla I, Sánchez-Payá J, Giner L, et al. Subclinical atherosclerosis in low framingham risk HIV patients. Eur J Clin Inves 2017; 47(8):591-9. doi: 10.1111/eci.12780.

23. Thompson-Paul AM, Lichtenstein KA, Armon C, Palella Jr FJ, Skarbinski J, Chmiel JS, et al. Cardiovascular disease risk prediction in the HIV outpatient study. Clin Infect Dis 2016; 63(11):1508-16. doi: 10.1093/cid/ciw615.

24. Markowicz S, Delforge M, Necsoi C, De Wit S. Cardiovascular risk evaluation of HIV-positive patients in a case-control study: Comparison of the $D: A: D$ and framingham equations. J Int Aids Society 2014; 17(4S3). doi: 10.7448/IAS.17.4.19515. 